\title{
Model of the influence of magnetic fields on a plasma electrode Pockels cell
}

\author{
C.D. Boley and M.A. Rhodes
}

This paper was prepared for submittal to the

Solid-State Lasers for Application to Inertial Confinement Fusion (ICF) 2nd Annual International

Conference

Paris, France

October 22-25, 1996

October 22, 1996

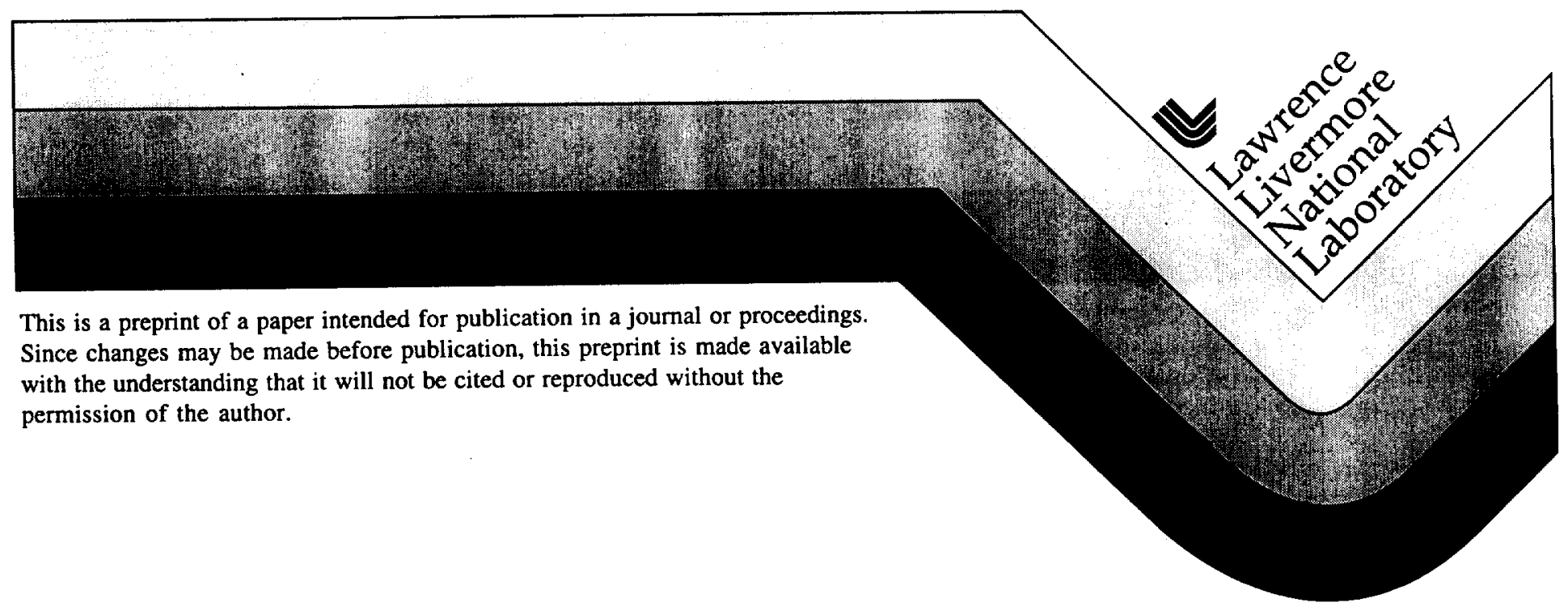




\section{DISCLAIMER}

This document was prepared as an account of work sponsored by an agency of the United States Government. Neither the United States Government nor the University of California nor any of their employees, makes any warranty, express or implied, or assumes any legal liability or responsibility for the accuracy, completeness, or usefulness of any information, apparatus, product, or process

disclosed, or represents that its use would not infringe privately owned rights. Reference herein to any specific commercial product, process, or service by trade name, trademark, manufacturer, or otherwise, does not necessarily constitute or imply its endorsement, recommendation, or favoring by the United States Government or the University of California. The views and opinions of authors expressed herein do not necessarily state or reflect those of the United States Government or the University of California, and shall not be used for advertising or product endorsement purposes. 
Model of the influence of magnetic fields on a plasma electrode Pockels cell

\author{
C. D. Boley and M. A. Rhodes \\ University of California \\ Lawrence Livermore National Laboratory \\ Livermore, CA 94550 USA
}

\begin{abstract}
We describe a model which gives the effects of magnetic fields on a plasma electrode Pockels cell. The fields arise from the return currents to the cathode as well as from neighboring devices such as amplifier flashlamps. In effect, electrons are treated as a static, planar fluid moving under the influence of magnetic fields, the electric field of the discharge, electron pressure gradients, and electron-atom elastic collisions. This leads to a closed two-dimensional equation for the electron density, which is solved subject to appropriate boundary collisions. The model is applied to four cases: the baseline NIF configuration with magnetic fields due to balanced return currents; a case with unbalanced return currents; the reverser configuration containing an external field parallel to the main plasma current; and a configuration with a field perpendicular to both the current and the optical direction.
\end{abstract}

\title{
1. INTRODUCTION
}

A plasma-electrode Pockels cell ${ }^{1.2}$ is a critical element of laser systems employing high-fluence multipass amplifiers, such as NIF. Used in conjunction with a polarizer, this device performs active switching of the laser beam. Thin, planar plasmas on either side of the crystal serve as electrodes. Magnetic fields can affect the behavior of these plasmas, leading to nonuniform charging of the crystal. Such fields can be produced by the return currents to the cathode, by the plasma current itself, and by currents from nearby devices such as amplifier flashlamps. We have constructed a model to describe the effects of these fields on the discharge. In essence, we treat the electrons as a planar fluid which responds to the magnetic field, along with forces from the electric field of the discharge, electron pressure gradients, and electron-atom elastic collisions. The geometry of the model is sketched in Fig 1. The wires to the cathode run above and/or below the plasma. Since opposing currents repel, we expect the electrons to be pushed away from these wires as they leave the cathode region. In the approximation of a planar plasma, the field due to the wires and the plasma current is perpendicular to the current sheet. Figure 2 shows a measured emission pattern which illustrates plasma pinching. In this case, the effect is due to fields set up by the return currents and the plasma current. After electrons leave the cathode region, they tend to flow toward the vertical center of the discharge, before eventually being collected at the anode. We have not attempted to deduce the actual electron density from the emission strength, but it is clear that pinching has the potential to prevent the crystal from charging uniformly.

Our model leads to a closed two-dimensional equation for the electron density, which is solved subject to appropriate boundary collisions. We consider four cases: the baseline NIF configuration with magnetic fields due to balanced return currents; a case with unbalanced return currents; the reverser configuration containing an external field parallel to the main plasma current; and a configuration with a field perpendicular to both the current and the optical direction.

\section{MODEL}

We employ a 2-D fluid model of the plasma, neglecting structure in the thin direction, parallel to the light. Since the observed patterns tend to change on time scales no faster than a microsecond or so (the time scale of the driving current), we consider the static limit. The continuity condition for electrons and ions is

$$
\nabla \cdot\left(n \mathbf{v}_{e}\right)=\nabla \cdot\left(n \mathbf{v}_{i}\right) \pm 0
$$



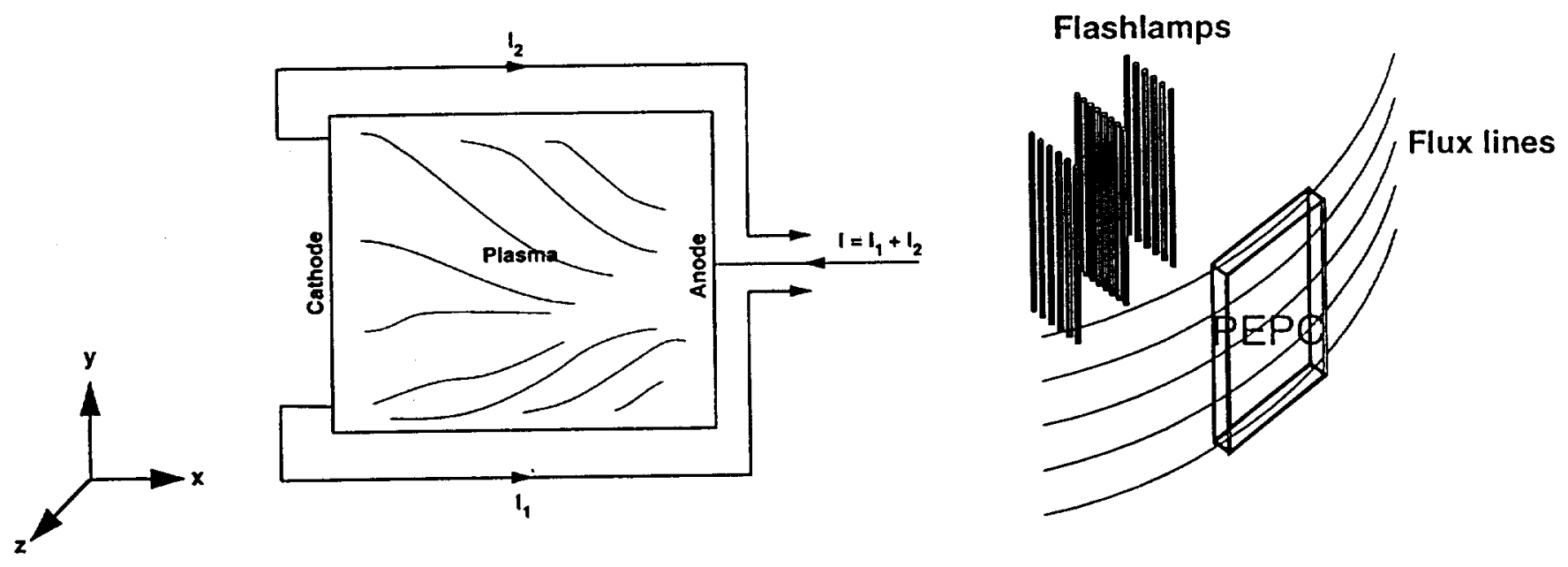

Fig.1. Geometry of the model.

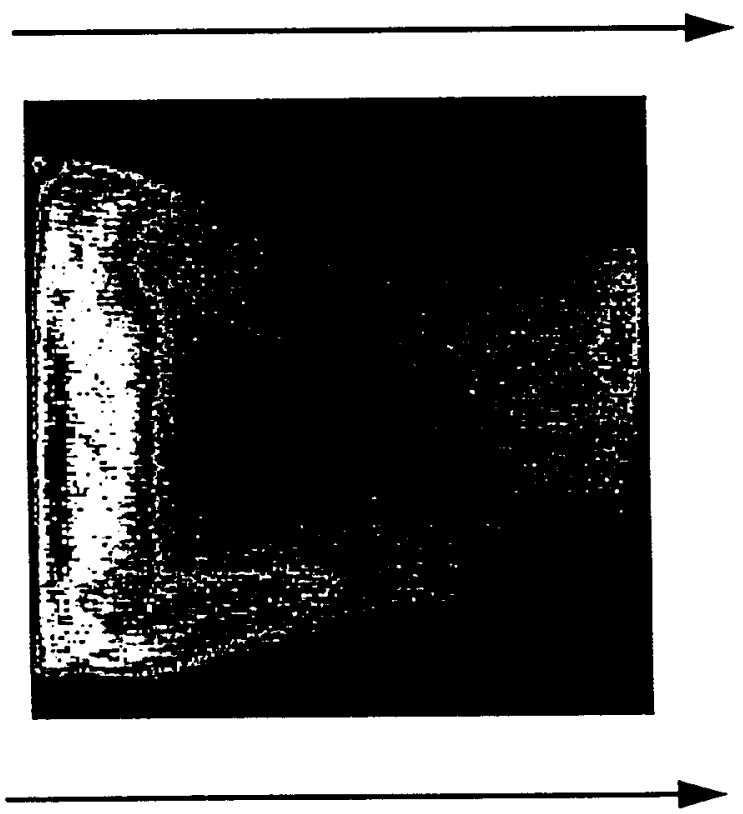

Fig. 2. Observed emission pattern at $447 \mathrm{~nm}$ from neutral He. The intensity gives a qualitative measure of the local electron density. The cathode is on the left, the anode on the right. 
where charge neutrality $\left(n_{\epsilon}=n_{i} \equiv n\right)$ has been assumed and the velocity fields are $\mathbf{V}_{e}$ and $\mathbf{v}_{i}$. The ionization source is absent since ionization is assumed to be balanced by ambipolar diffusion in the perpendicular (z) direction ${ }^{3}$. This balance determines the electron temperature $T_{e}$, which is close to $5 \mathrm{eV}$ for typical operating conditions ${ }^{3}$. The electron and ion velocity fields satisfy

$$
\begin{gathered}
m n\left(\mathbf{v}_{e} \cdot \nabla\right) \mathbf{v}_{e}=-\nabla p_{e}-e n \mathbf{v}_{e} \times \mathbf{B}-e n \mathbf{E}-m n v_{e} \mathbf{v}_{e}, \\
M n\left(\mathbf{v}_{i} \cdot \nabla\right) \mathbf{v}_{i}=e n \mathbf{E}-M n v_{i} \mathbf{v}_{i}
\end{gathered}
$$

where $p_{e}=n T_{e}$ is the electron pressure, $v_{e}=n_{0}<\sigma v>$ is the momentum transfer frequency for collisions of electrons with the background gas (helium), and $V_{i}$ is the charge exchange frequency. Ion pressure and magnetization have been neglected. The electronic charge is $-e$. Because of the large electron thermal conductivity, the electron temperature has been taken as uniform.

Under the reasonable assumptions that the scale length of the density gradient is a few centimeters, that the magnetic field is of the order $10 \mathrm{G}$ (appropriate for currents in the kiloampere range), and that the electron velocity is near the thermal speed, one can check that the terms on the right-hand side of the electron momentum equation have about the same order of magnitude. However, the left-hand side, describing convection, is smaller than the other terms by an order of magnitude, at least in the situations modeled thus far. Similar considerations show that ion convection is not important. Thus we neglect convection in the momentum equations, verifying a posteriori that this is valid. We solve for the current densities and insert the results into the density conservation equations. From the electron momentum equation, the electron current density in the $x-y$ plane can then be expressed as

$$
J_{i}^{e}=\sum_{j} C_{i j}\left(\frac{\partial n}{\partial x_{j}}-\frac{e n}{T_{e}} \frac{\partial \phi}{\partial x_{j}}\right)
$$

where $i$ and $j$ are Cartesian indices in the plane, $\phi$ is the electric potential, and the matrix $C$ is

$$
C=\frac{e T_{e}}{m v_{e}\left(1+b^{2}\right)} \cdot\left(\begin{array}{cc}
1+b_{x}^{2} & b_{x} b_{y}-b_{z} \\
b_{x} b_{y}+b_{z} & 1+b_{y}^{2}
\end{array}\right) \text {. }
$$

The dimensionless vector $\mathbf{b}=e \mathbf{B} / m v_{e}$ is the ratio of the local gyrofrequency to the electron-neutral collision frequency. Its absolute maximum value typically lies between 1 and 10 . Similarly, the ion current density is just $J_{i}^{i o n}=-\left(e^{2} n / M v_{i}\right) \partial \phi / \partial x_{i}$. The resulting system, given by the continuity conditions applied to Eqs. (4) and (5), consists of a pair of two-dimensional partial differential equations in two unknowns -- the electron density and the potential.

Investigating approximate solutions of this system, we have noticed that the electric field turns out to be nearly uniform and straight (pointing from anode to cathode). This is reasonable on physical grounds. In the present discussion we take advantage of that simplification. Thus we take $\phi=-x E_{x}$, with $E_{x}$ the prescribed (negative) voltage across the discharge. Typically, the absolute value of the field is about 25 volts. This is much smaller than the full potential between the electrodes, since the latter is dominated by the cathode fall voltage of a few hundred volts. The model is not intended to describe cathode effects, which are confined to a few centimeters of the surface. In our approximation, then, the ion current responds merely to the impressed electric field, and we are concerned only with the electron current, as given by Eqs. (4) and (5). According to this system, the current density consists of a term proportional to the electric field and another term proportional to the electron density gradient. The former term corresponds to Ohm's law as modified by the magnetic field, and the latter term arises from the pressure gradient.

Inserting the electron current into the continuity equation, we obtain the following elliptic equation for the electron density: 


$$
\frac{\partial}{\partial x}\left[\frac{1+b_{x}^{2}}{1+b^{2}}\left(\frac{\partial n}{\partial x}+\frac{n e E_{x}}{T_{e}}\right)+\frac{b_{x} b_{y}-b_{z}}{1+b^{2}} \frac{\partial n}{\partial y}\right]+\frac{\partial}{\partial y}\left[\frac{b_{x} b_{y}+b_{z}}{1+b^{2}}\left(\frac{\partial n}{\partial x}+\frac{n e E_{x}}{T_{e}}\right)+\frac{1+b_{y}^{2}}{1+b^{2}} \frac{\partial n}{\partial y}\right]=0
$$

The boundary conditions on the sides without electrodes (the top and bottom) are that no current leaves, i.e. that the second term in square brackets above vanishes. At the cathode, or rather just beyond the magnetron region, the appropriate boundary condition evidently is a prescribed horizontal current density $J_{x}^{e}(y)$, which is proportional to the first term in square brackets. It might also seem possible to prescribe the current density at the anode, but this turns out not to be mathematically feasible, for reasons which are currently under study. Instead we have simply imposed a zero horizontal gradient on the electron density at the anode, thus smoothing features, as a function of $x$, at this end.

\section{APPLICATIONS}

We now turn to four examples which illustrate the predictions of the model. The self field of the plasma is not included, as it is still in the process of being implementing. This field would lead to increased pinching.

\subsection{Magnetic field due to return currents: balanced currents}

In the first example, we consider a cell of dimensions $45 \mathrm{~cm}$ (horizontally) $\times 40 \mathrm{~cm}$ (vertically), operating under typical conditions. The return current of $2 \mathrm{kA}$ is divided evenly between wires running above and below the cell. The horizontal segment of each wire is displaced $10 \mathrm{~cm}$ from the cell, and fringing effects are taken into account.

In Fig. 3(a) contours of the magnetic field due to the wires are plotted. The normal electron current leaving the cathode is assumed to be uniformly distributed along the cathode. Thus the absolute value of the magnetic field increases near the cathode due to fringing. Figure 3(b) shows contours of the resulting electron density, displaying the pinching produced by the external currents. Typically, the density at a distance a few centimeters from the top and bottom edges is lower than that along the horizontal centerline by about a factor of 9 . For proper charging of the crystal, only a minimum electron density is required ${ }^{3}$.

This pinching is also evident in the plasma current pattern, shown in Fig. 4. Since the external conditions are vertically symmetrical, the patterns are also symmetrical.

\subsection{Magnetic field due to return currents: unbalanced currents}

Next we consider the situation in which the total current remains the same but the split between top and bottom wires is 2:1. Now the field becomes dominant in the top half, as seen in Fig. 5(a). The calculated density contours, shown in Fig. 5(b), are shifted toward the bottom, as expected. Near the top, the density has fallen by an order of magnitude relative to the previous case, indicating that switching may be degraded. The plasma current density, displayed in Fig. 6, shows a similar diversion toward the bottom of the cell.

\subsection{Magnetic field parallel to main plasma current}

We now turn to a case relevant to the proposed reverser experiment, in which an amplifier module is placed close to the Pockels cell such that the field from the flashlamps intercepts the cell primarily along the cathode-anode $(x)$ direction. Its strength is of the order $20 \mathrm{G}$. Since this field is parallel to the plasma current, we expect it to oppose the pinching effect observed above. Thus it should have a beneficial effect on discharge uniformity. This is born out in the results (Fig. 7) which show that the electron density contours have opened up relative to those of the first example. Half way across the discharge, for example, the density now varies by about a factor of about 3 from horizontal centerline to edge. 

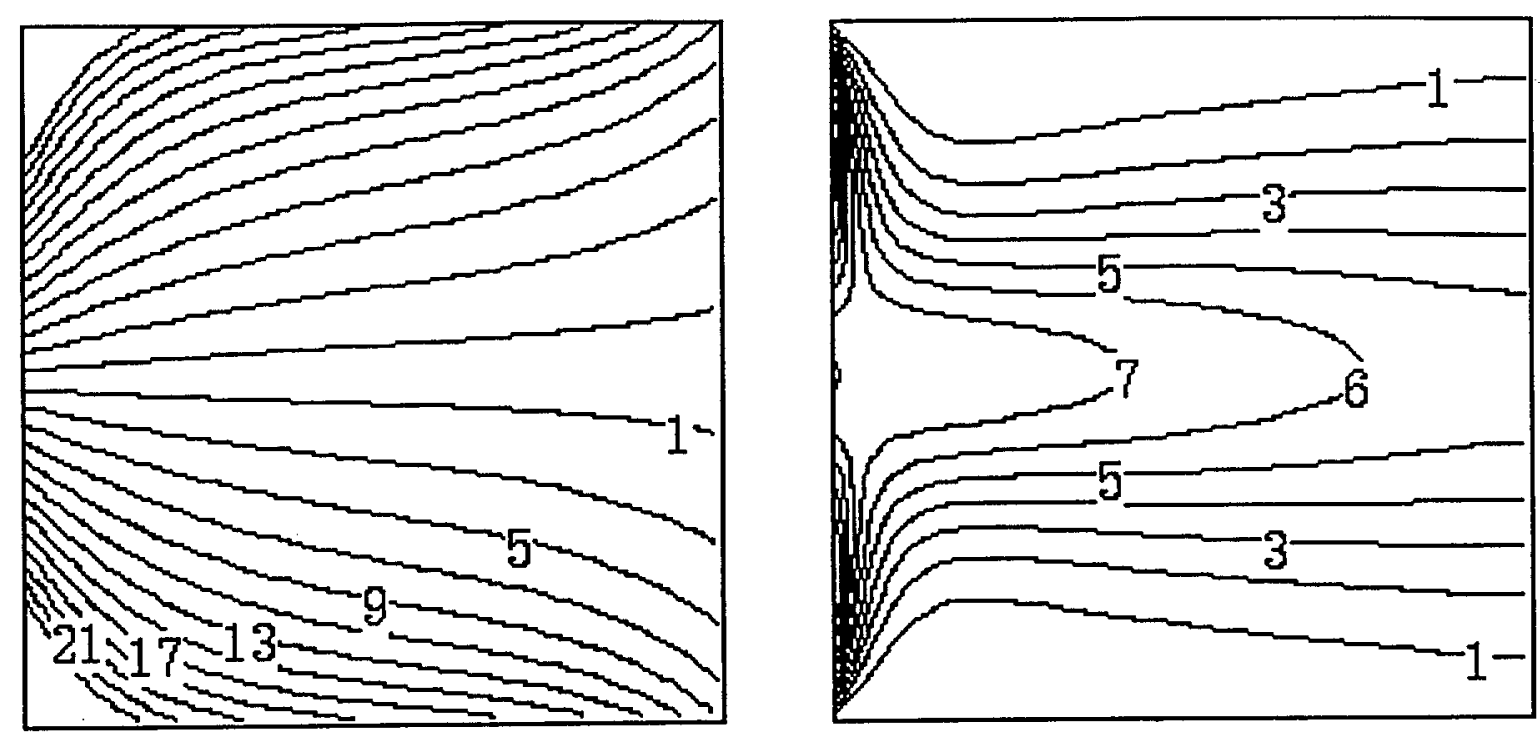

Fig. 3. (a) Magnetic field (in Gauss), for the case in which the current returns carry equal currents of $1 \mathrm{kA}$. The field is odd with respect to the horizontal centerline, so that the upper values are negative. (b) Electron density contours for this case, in units of $10^{12} \mathrm{~cm}^{-3}$.

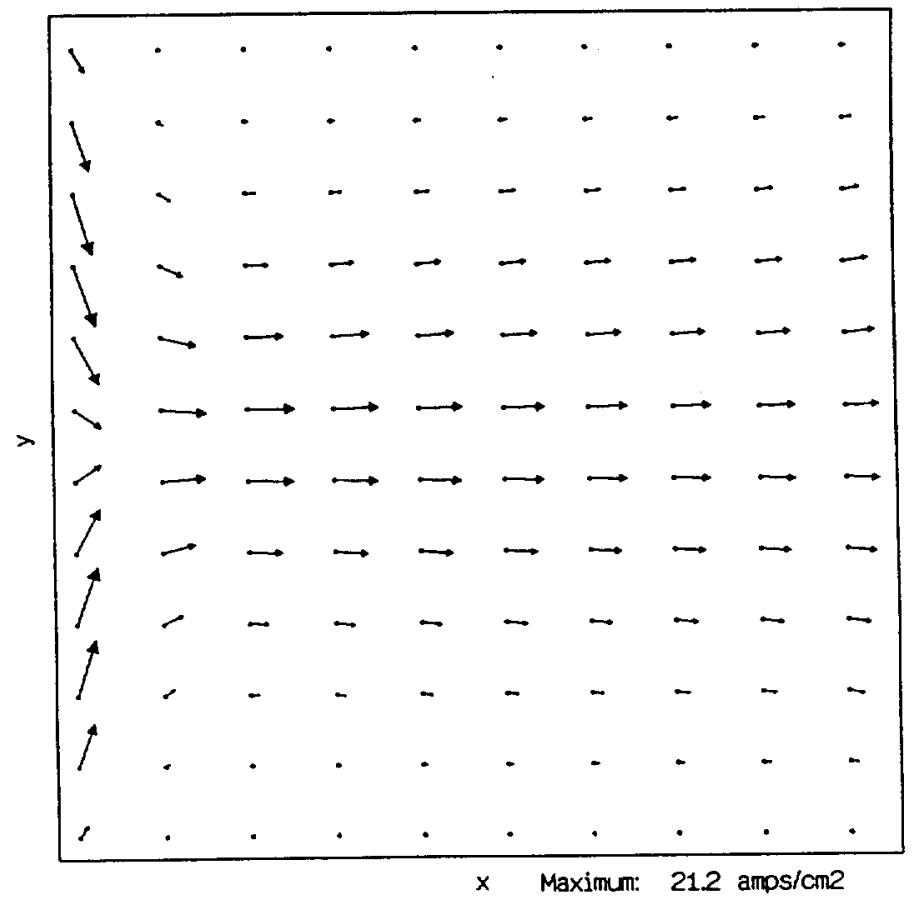

Fig. 4. Plasma current density for the case of equal current returns. The arrows indicate -J. 

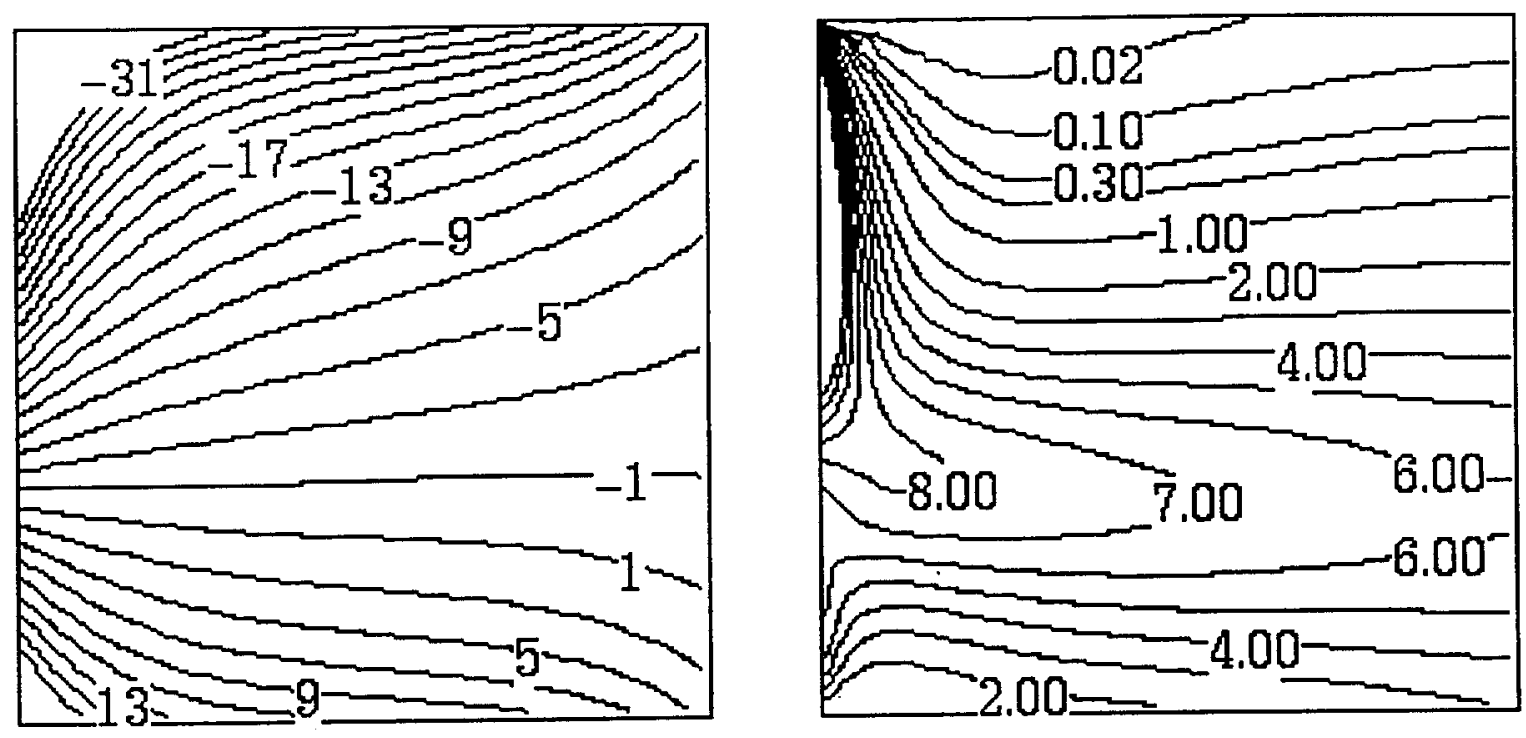

Fig. 5. (a) Magnetic field, for the case in which the top and bottom return currents carry $1333 \mathrm{~A}$ and $667 \mathrm{~A}$, respectively. (b) Electron density contours. Units are the same as in Fig. 3.

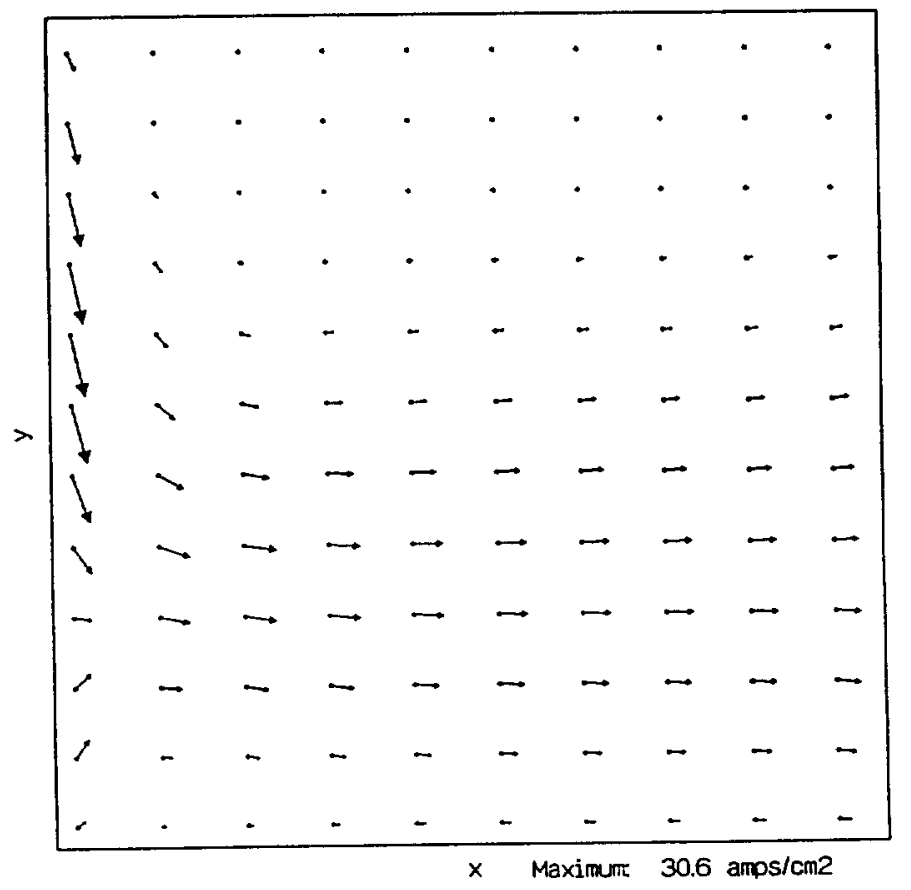

Fig. 6. Plasma current density patterns, for the case of unequal current returns. 


\subsection{Magnetic field perpendicular to both current and light}

In this configuration, the magnetic field lies within the plane of the cell, as in the previous example, but it is rotated so that it is perpendicular to the main plasma current. If there were a second amplifier bank in NIF, then the field from its flashlamps would intercept the cell in this manner, since the cathode-anode axis is vertical. Such a field diverts the plasma current toward the sides of the cell, i.e. toward either the window or the crystal, depending on which of the two plasmas is under consideration. Hence it can be deleterious to switching performance. Since this flow pattern is not confined to the plane, it nominally requires a 3-D model. However, we can adapt our 2-D model in an approximate sense by viewing the plasma from above, as illustrated in Fig. 8. The $x$-axis corresponds to the cathode-anode direction, as before, but now the $y$ axis lies in the optical direction. The external magnetic field is perpendicular to this plane, as required by the model. We are forced to neglect the magnetic field set up by the return currents, since it lies in the $y$-direction. With this modification of the model, though, we can get a rough idea of the effects of the external field.

Simulations show that, sufficiently far from the cathode, the electron density becomes stratified in the optical direction and becomes nearly uniform in the cathode-anode direction. Thus the plasma on one side of the cell tends to collect at the window, degrading the switch performance. This pattern has a simple analytical form. In the governing equation (6), with $\mathrm{B}=\mathrm{B}_{0}=$ constant, we look for a solution in which the electron density depends only on $\mathrm{y}$. In this situation, the $\mathrm{JxB}$ force is balanced by the electron pressure gradient, and the electric field is balanced by the electron-atom friction term. Of course such a form cannot be valid near the cathode, where the current is uniform in y. However, it becomes accurate a few centimeters from the cathode. The solution is

$$
n(y)=\frac{I B_{0}}{h T_{c}} \exp (\kappa y) /[\exp (\kappa w)-1],
$$

where $I$ is the total current, $h$ is the height of the discharge, $w$ is the width of the discharge (from window to crystal), and $\kappa=-e^{2} B_{0} E_{x} / m v_{e} T_{e}>0$. Note that the electron density increases exponentially with $y$. The velocity field is uniform, so that the current density is proportional to the electron density. The density mismatch from side to side in the thin direction, $n(w) / n(0)$, increases exponentially with the magnetic field. Under typical conditions $(h=40 \mathrm{~cm}, w=5 \mathrm{~cm}, I=2000$ A), the density mismatch equals about 3 for a field of $10 \mathrm{G}$ and increases to 10 when the field is doubled. Therefore a $10 \mathrm{G}$ field might possibly be tolerable for switch operation, but a $20 \mathrm{G}$ field is clearly too large.

\section{CONCLUSIONS}

We have constructed a model which describes the effects of magnetic fields on a plasma electrode Pockels cell. Treating the electrons as a static, planar fluid, we have found the governing equation for the electron density, which we have solved subject to appropriate boundary conditions. The model has been applied to four cases: the NIF configuration with magnetic fields due to balanced current returns, a similar case with unbalanced current returns, the proposed reverser configuration with an external field parallel to the main plasma current, and a configuration characterized by a field perpendicular to both the current and the optical direction. In all cases, the model appears to give reasonable predictions. Calculations are currently being extended to include effects such as the field generated by the plasma current.

\section{ACKNOWLEDGMENTS}

We would like to thank B. S. Bauer, A. C. Hindmarsh, W. A. Molander, and J. B. Trenholme for useful discussions. This work was performed under the auspices of the U. S. Department of Energy by the Lawrence Livermore National Laboratory under contract number W-7405-ENG-48.

\section{REFERENCES}

1. J. Goldhar and M. A. Henesian, "Electro-Optical Switches with Plasma Electrodes," Optics Letters 9, 73-75 (1984).

2. M. A. Rhodes, B. Woods, J. J. DeYoreo, D. Roberts, and L. J. Atherton, "Performance of Large-Aperture Optical Switches for High-Energy ICF Lasers," Applied Optics (1995).

3. M. A. Rhodes, C. D. Boley, A. G. Tarditi, and B. S. Bauer, "Plasma Electrode Pockels Cell for ICF," 1st Annual International Conference on Solid State Lasers for Application to ICF, SPIE 2633, 1995. 


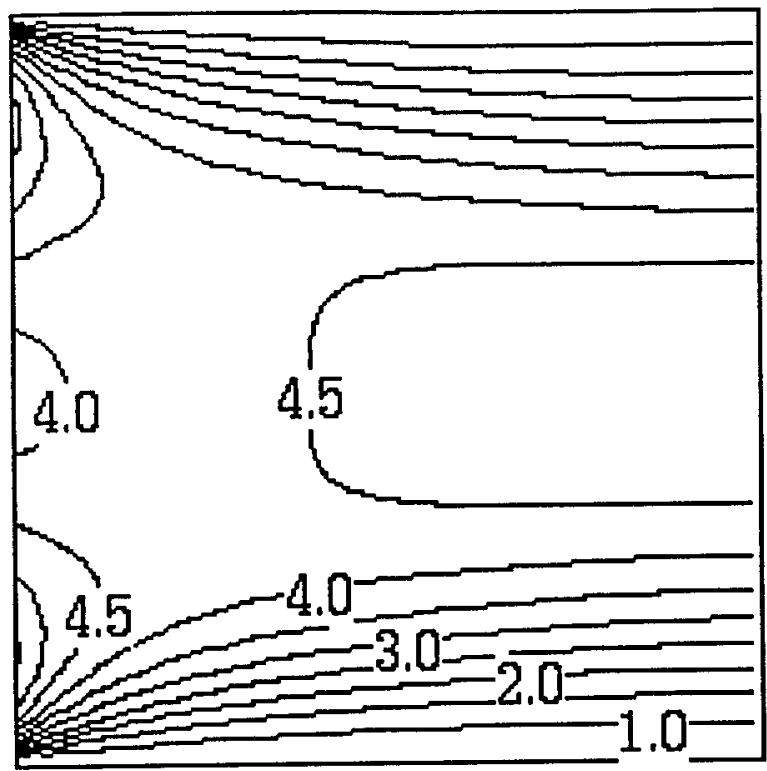

Fig. 7. Electron density contours (units of $10^{12} \mathrm{~cm}^{-3)}$ for the case of an external field parallel to the main plasma current (balanced current returns of $1 \mathrm{kA}$ on both top and bottom).

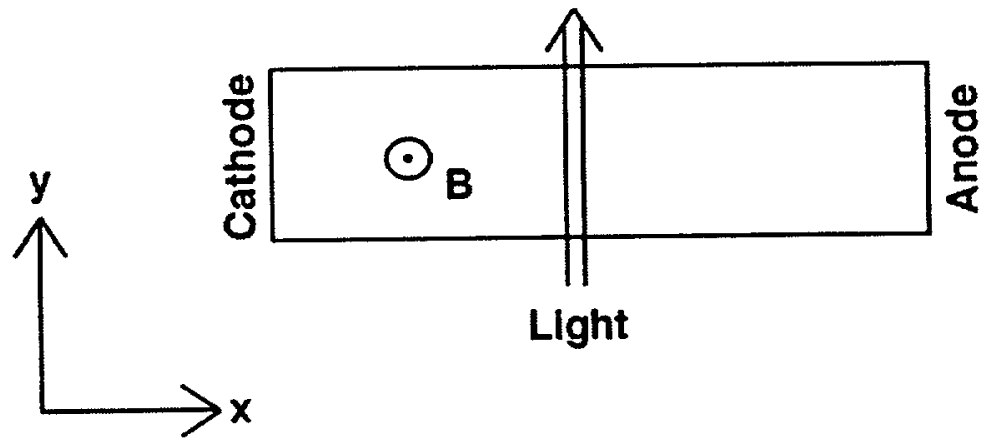

Fig. 8. Geometry for the case of a magnetic field perpendicular to both the main plasma current and the optical direction. 


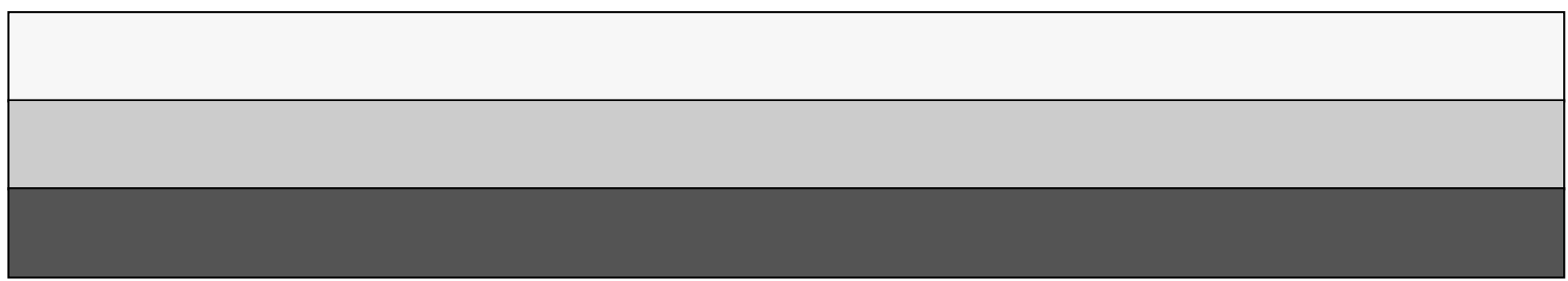

